\title{
Querying XML sources using an Ontology-based Mediator
}

\author{
Bernd Amann $^{1}$, Catriel Beeri ${ }^{\star 2}$, Irini Fundulaki ${ }^{1}$, and Michel Scholl ${ }^{1}$ \\ 1 Cedric-CNAM Paris and INRIA-Futurs, France \\ amann@cnam.fr, fundulak@cnam.fr, schollecnam.fr \\ ${ }^{2}$ The Hebrew University, Jerusalem, Israel \\ beeri@cs.huji.ac.il
}

\begin{abstract}
In this paper we propose a mediator architecture for the querying and integration of Web-accessible XML data sources. Our contributions are (i) the definition of a simple but expressive mapping language, following the local as view approach and describing XML resources as local views of some global schema, and (ii) efficient algorithms for rewriting user queries according to existing source descriptions. The approach has been validated by the $S T_{Y} X$ prototype.
\end{abstract}

\section{Introduction}

During the last decade, there has been a significant focus on data integration. In a nutshell, data integration can be described as follows: given heterogeneous and autonomous information sources in a specific domain of interest, the goal is to enable users to query the data as if it resides in a single source, with a single schema. To achieve this goal, a global schema of the data is defined, and related to the schemas of the individual sources. Queries are formulated in terms of this global schema. Since the actual data resides in the sources, queries are rewritten into queries over the source schemas, which are then evaluated at the sources. The answers returned from the sources are combined, transformed to be compatible with the global schema, and presented to the user. The integration facilities, namely the global schema, the query translation and query processing algorithms, are performed by a mediator, whose main task is to provide users with a unique interface for querying the data. The fact that the sources concern a restricted domain of interest, is crucial for the successful deployment of integration systems.

Well-known projects that deal with data integration include Information Manifold [12], Tsimmis [14], Picsel [10], Agora [13] and MIX [3]. As the goal of integration is to support declarative querying and automatic query and result transformations, a number of data integration systems use the well-established tools available for such purposes in the relational model, such as query and transformation languages.

Recently, XML [1] has emerged as the de-facto standard for publishing and exchanging data on the Web. Many data sources export XML data, and publish their contents using DTD's or XML schemas. Thus, independently of whether the data is actually stored in XML native mode or in a relational store, the view presented to the users is XML-based. The use of XML as a data representation and exchange standard raises

\footnotetext{
${ }^{\star}$ Research supported by grant $018-019$ by the Israeli Ministry of Science.
} 
new issues for data integration. A significant issue, as argued in [2], is the inadequacy of XML to serve as a global integration schema.

In this paper we describe an approach to the integration of XML sources, based on the local-as view [11] approach to data integration. Our main contributions are as follows: (i) the use of ontologies for the global schema; (ii) the definition of a simple but expressive language for describing XML resources as views of the global schema; (iii) an approach to query processing, that includes query rewriting from the terms of the global schema into one or more XML queries over the local sources, and (iv) the generation of query execution plans that may decompose a single query into queries over multiple sources. The approach has been validated by the $S T_{Y} X$ prototype [9].

The paper is organized as follows : in Section 2 we illustrate the main ideas of the approach by an example. Section 3 presents the integration data model, and the mapping language for the description of XML resources as views over the global schema. The query language, and the query processing algorithms are given in Section 4 . The $S T_{Y} X$ prototype is sketched in Section 5. Related work is presented in Section 6, and Section 7 presents our conclusions.

\section{System Overview}

We illustrate our approach via an example dealing with the integration of XML-based information sources on art and culture. Formal definitions and technical details are deferred to subsequent sections.

\subsection{XML resources}

Source $S_{1}$, located at http://www.paintings.com is an XML resource about painters and their paintings; its XML DTD is illustrated in Fig. 1.

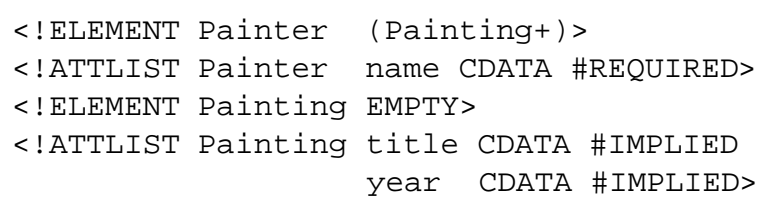

Fig. 1. XML DTD for source $S_{1}$, located at URL http://www.paintings.com

The XML DTD for the second source $S_{2}$, located at URL http://www.art.com, is described in Fig. 2.

As is common in data integration scenarios, a single source may provide only part of the information available on a subject. Furthermore, sources differ not only in terms of contents, but also in terms of structure and terminology. Given the hierarchical structure of XML, such differences of structure may be more significant that those that exist in relational sources. For an example of a difference of contents, note that source $S_{2}$ might record information on the location of paintings, which is absent in source $S_{1}$. As for structure, note that in source $S_{2}$ paintings are organized by museums, not by their 


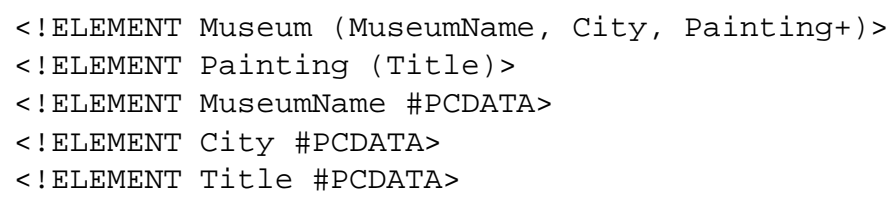

Fig. 2. XML DTD for source $S_{2}$, located at http://www.art.com

painters as in source $S_{1}$. Consequently, while in the hierarchy of $S_{1}$ a painting occurs below its painter, in source $S_{2}$, if the source were interested in adding the painter for each painting, the painter would occur below the painting.

\subsection{The Global Schema}

The main task of an integration mediator is to provide users with a unique interface for querying the data, independently of its actual organization and location. In our approach, this interface, or global schema, is described as an ontology. As used here, an ontology denotes a light-weight conceptual model and not a hierarchy of terms or a hierarchy of concepts.

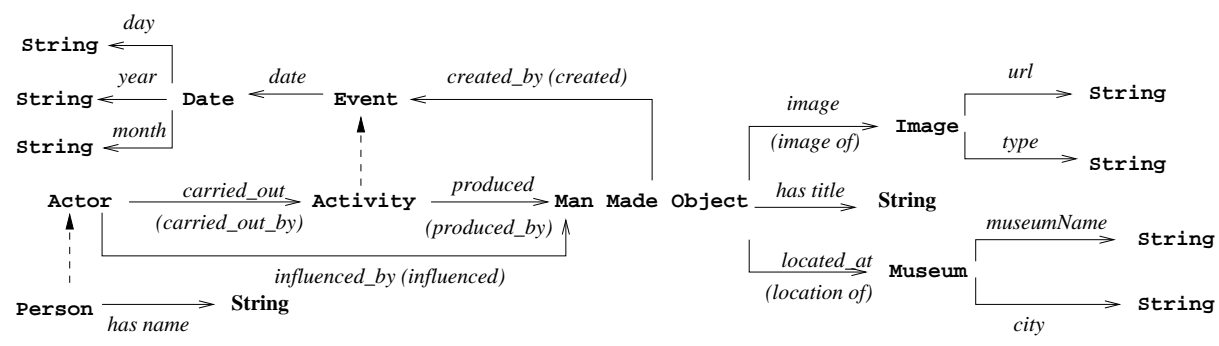

Fig. 3. An Ontology for Cultural Artifacts

Fig. 3 illustrates (part of) a global schema for cultural artifacts inspired by the ICOM/CIDOC Reference $\mathrm{Model}^{3}$, an international standard for museum documentation. The schema is represented as a labeled graph. In this graph, the nodes correspond to concepts and value types, and the edges depict roles, attributes, and simple inheritance (i.e., isa) links. Roles are binary relations between concepts; attributes connect concepts to value types. Both are depicted by solid arcs. Inheritance (isa) links connect concepts and are depicted by dashed arcs. Each role has an inverse depicted in Fig. 3 within parentheses.

The concepts in this schema include Actor, its subconcept Person, and Man Made_Object. An actor (instance of concept Actor) carries out an activity (instance of concept Activity) to produce a man made object (instance of concept Man Made_Object).

\footnotetext{
${ }^{3}$ http://cidoc.ics.forth.gr/crm_intro.html
} 
These relationships are represented by roles carried sut and produced, respectively. The name of a person (instance of concept Person) is represented by the attribute has_name.

The global schema can be viewed as a simple object-oriented data model. Hence, a global schema can be viewed as defining a database of objects, connected by roles, with the concept extents related by subset relationships as per the $i s a$ links in the schema. Since it is an integration schema, this is a virtual database. The actual materialization exists in the sources.

Roles can be composed, provided they satisfy certain compatibility constraints. Such compositions are derived roles. For example, carried sut.produced is a derived role that connects Actor to Man_Made_Object. Combining concepts with (simple or derived) roles induces derived concepts. For example, Actor.carried sut.produced can be viewed as the sub-concept of Man_Made_Object made of those objects that are reachable from some actor by an instance of this derived role. Both derived roles and derived concepts are referred to as schema paths.

The augmentation of the given schema with the derived roles and concepts gives a derived schema. It is significant for the integration, since it provides an interpretation for the mapping rules (see the following) that describe the sources in terms of schema paths, hence for query processing, as discussed next.

\subsection{Mapping Rules}

Our integration approach describes XML sources as local views on the global schema. Among the different possibilities listed in [7] for defining such mappings, we have chosen the path-to-path approach. The description of a source consists of mapping rules that associate paths in the source DTD, expressed in XPath [6], with paths in the global schema (schema paths). For example, the rules illustrated in Fig. 4 map paths in the source $S_{1}$ described in Fig. 1 to paths in the global schema of Fig. 3.

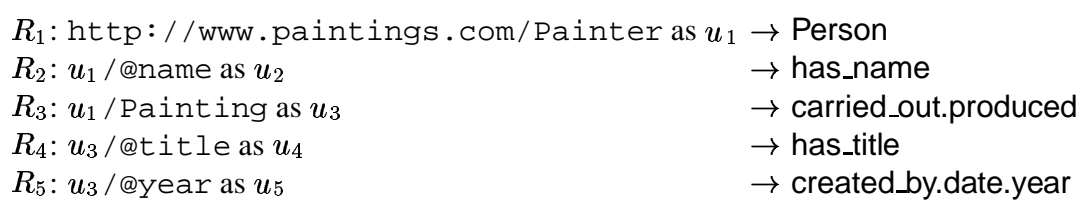

Fig. 4. Set of Mapping Rules for source http://www.paintings.com

A rule consists of a name, a left hand side (LHS) and a right hand side (RHS). The LHS contains an XPath pattern [6] that starts at a context which is either a concrete URL, as in rule $R_{1}$ or a variable, as in rule $R_{2}$. The XPath pattern is called the location path of the rule. The LHS of a rule also contains a variable declaration (the use of variables will be explained later). The RHS of a mapping rule is a path in the global schema, called the schema path of the rule.

Mapping rules define instances of concepts and relationships between them. As an example for the first case, consider the rule $R_{1}$ in Fig. 4. It states that the elements 
of type Painter, children of the root elements of the XML documents in $S_{1}$ are (descriptions of) instances of concept Person. As an example for the second case, rule $R_{2}$ specifies that the value obtained by evaluating XPath pattern @ name on some XML element $x$ returned by rule $R_{1}$, corresponds to a value of attribute has name of $x$ ( $x$ is an instance of concept Person). In the same way, rule $R_{3}$ connects all instances obtained by rule $R_{1}$ to all instances of concept Man_Made_Object obtained by following the path carried_out.produced.

This view of mapping rules allows us to define the semantics of XML fragments and their structural relationships in terms of the global derived schema. Thus, $R_{1}$ defines a subset of the extent of concept Person, while rule $R_{3}$ relates elements in this subset by the derived role carried_out.produced to a subset of the extent of Man Made_Object.

\subsection{Query Processing}

Users formulate queries on the global schema using a simplified variant of OQL, the standard for querying object databases. For example, here is a query $Q_{1}$ that asks for "titles of the man made objects created by Van Gogh":

$Q_{1}$ : select $c$

from Person $a, a$.has_name $b$, a.carried_out.produced.has _title $c$

where $b=$ "Van Gogh"

We now discuss the options available for answering such a query, given a set of sources $S$ and mapping rules that relate them to the global schema.

The first, simple, solution is to evaluate this query over each source in $S$. This means that, given a source $s \in S$, we need to rewrite it into an XML query that $s$ can answer. The idea behind this rewriting is the following: Each variable in the query is bound to some schema path. We search for mapping rules or concatenations of mapping rules, that can be used to translate these schema paths to local paths in the source DTD. This is done by matching the schema paths in the query against the schema paths of the mapping rules. A successful matching associates a query variable with a rule, or a concatenation of rules. A binding is a vector of such associations for query variables. A full binding associates each variable in the query to some rule or concatenation of rules. It can be used to rewrite the query into a query to be evaluated by the XML source.

For example for $Q_{1}$ above and for source $S_{1}$ we see that instances for variable $a$ are found by rule $R_{1}$, for variable $b$ by $R_{2}$ and for variable $c$ by the concatenation of rule $R_{3}$ with rule $R_{4}$. The resulting binding is $\left[a \rightarrow R_{1}, b \rightarrow R_{2}, c \rightarrow R_{3} . R_{4}\right]$. By substituting the schema path of each query variable with the location path (LHS) of the corresponding rule, we obtain query $Q_{1}(a)$ :

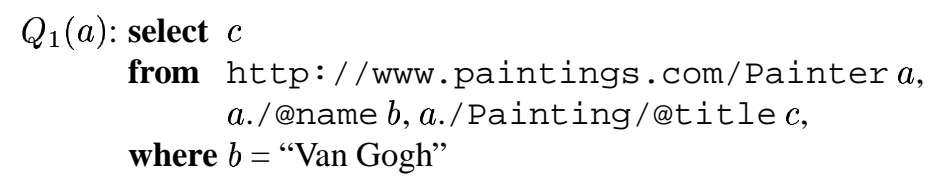

Query $Q_{1}(a)$ can be easily translated into the XQuery expression $Q_{1}(b)$ : 


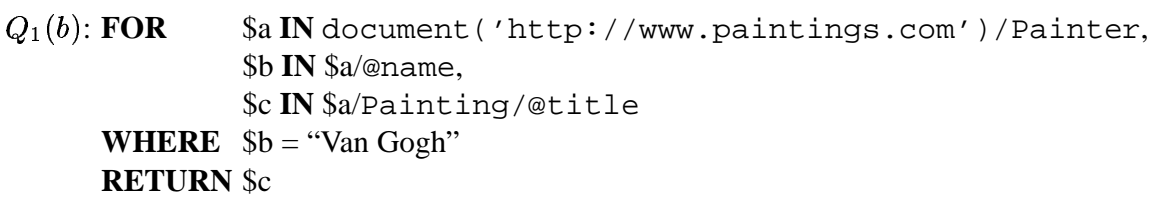

Such a matching/rewriting process should be attempted for each source. Then the answers are gathered and returned to the user.

In some cases, however, we cannot obtain a full binding for a given source. Then a second solution for query evaluation is to decompose the query into several queries that are evaluated against different sources. Consider the following query $Q_{2}$, which asks for "titles of objects created by Van Gogh, as well as the name and the city of the museum where they are exposed":

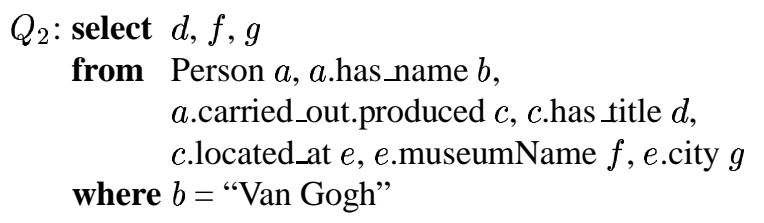

Source $S_{1}$ cannot provide information about the locations of objects, hence there is no mapping rule whose schema path (RHS) matches the schema path located at of query variable $e$. Thus, we can only obtain a partial answer from this source, by evaluating the query $Q_{2}(a)$ illustrated in Fig. 5. To obtain a full answer we have to join partial answers from different sources.

For the example, the missing information is represented by subquery $Q_{2}(b)$ illustrated also in Fig. 5, that involves the variables $c, e, f, g$. The variable $c$ is included in $Q_{2}(b)$ since it is the join variable between the two queries. Thus, we have decomposed the initial query into two subqueries $Q_{2}(a)$ and $Q_{2}(b)$. Assuming the latter query is successfully evaluated over some source (e.g., source $S_{2}$ ), the results of the two queries are joined on $c$ to provide a complete answer to the original query. If such a decomposition cannot be found, the best we can do is to present to the user only the partial results from $Q_{2}(a)$ evaluated against the first source.

Note that to join two fragments from different sources requires to decide whether the two fragments represent identical objects. Keys are introduced to identify objects. In particular, results of queries $Q_{2}(a)$ over a source $S_{1}$ and of $Q_{2}(b)$ over a source $S_{2}$ can be joined only if the same key for man made objects can be provided by these two sources. This implies the use of keys, both in the global schema and in the sources (the DTD's in Fig. 1 and Fig. 2 do not define such keys). We introduce keys and their usage in Section 3.2.

\section{Integration Model}

This section is devoted to the detailed presentation of our integration model. Due to space limitations we leave out a detailed discussion concerning the choices of the integration method, and the choice of having a light weight conceptual schema for the mediator schema instead of an XML-based model. A detailed presentation of these 


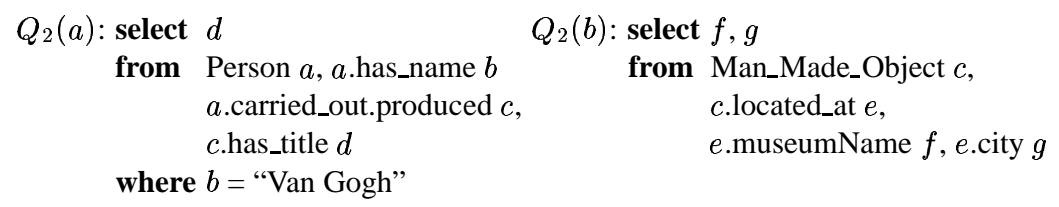

Fig. 5. Queries $Q_{2}(a)$ and $Q_{2}(b)$

choices is given in [2]. We first provide a formal definition of the global schema and introduce the notion of derived schema. Mapping rules are described afterwards and we finish this section with a short discussion on keys.

\section{Global Schemas}

A global schema is a 6-tuple $\mathcal{S}=(C, R, A, i s a$, source,target), where: (i) $C$ is a set of concepts, (ii) $R$ is a set of typed binary roles connecting concepts in $C$, (iii) $A$ is a set of attributes of type String ${ }^{4}$, (iv) $i s a$ is a binary relationship between concepts in $C$, (v) source and target are two typing functions returning for each role/attribute its domain concept and its range concept/type respectively.

A global schema can be represented as a graph of concepts connected by roles. The semantics of a schema is defined by the set of databases that conform to it. Each such database contains a set of objects (instances) for each concept in $C$. These objects are related to each other by instances of roles in $R$, and to values by instances of attributes in $A$. Instances of roles and attributes satisfy the typing constraints implied by source and target. Roles and attributes are multi-valued and optional. The $i s a$ relationship defines a partial order in $\mathcal{S}$, namely a directed acyclic graph. It carries subset semantics and supports role and attribute inheritance. Namely, if $c$ is a $c^{\prime}$, then the set of objects of $c$ is a subset of the set of objects of $c^{\prime}$ and all roles/attributes defined in $c^{\prime}$ are also defined in $c$ (and its subconcepts). However, if $c$ is not a subconcept of $c^{\prime}$ such that source $(r)=c^{\prime}$ for a role $r$ or source $(a)=c^{\prime}$ for an attribute $a$, then no object $o^{\prime}$ of $c$ is related by an instance of $r$ or $a$ to any object, or value respectively. We say that $c, c^{\prime}$ are isa-related if either $c=c^{\prime}$, c isa $c^{\prime}$ or $c^{\prime}$ isa $c$.

Finally, we consider schema graphs to be symmetric : each role $r \in R$ has an inverse role, denoted $r^{-}$, in $R$. Obviously, $\operatorname{target}\left(r^{-}\right)=\operatorname{source}(r)$, and $\operatorname{source}\left(r^{-}\right)=$ target $(r)$. This is useful for modelling the contents of XML resources as well as for query formulation and, hence, beneficial to have in a conceptual schema.

Schema Paths and Derived Schemas We distinguish two kinds of paths in a schema:

- A role path is a sequence of roles $r=r_{1} \ldots r_{n}$, where for all roles $r_{i}(1 \leq i \leq$ $n-1)$, target $\left(r_{i}\right)$ is a source $\left(r_{i+1}\right)$. Given a role path $r=r_{1} \ldots r_{n}$, we define its inverse role path $r^{-}=r_{n}^{-} \ldots r_{1}^{-}$where $r_{i}^{-}$is the inverse role of $r_{i}$

\footnotetext{
${ }^{4}$ Wlg. we assume that all attributes are of type string; an extension to the types proposed by the XPath model or XML schema should be straightforward.
} 
- A concept path $p$ is either of the form $c$, or a sequence $c . r$, where $c$ is a concept and $r$ is a role path, such that $c$ isa source $(r)$. The source of $p$ is $c$ and its target is $c$ in the first case, and target $(r)$ in the second case.

The composition of a concept path $p$ and a role path $r$, denoted $p \circ r$, is well-defined provided that target $(p)$ isa source $(r)$.

A concept path $p=c . r$ can be viewed as a derived concept (denoted by conc $(p)$ ), standing for "the instances of target $(p)$ that can be reached from instances of source $(p)$ by following the roles in $p$, in order". Obviously, every concept is also a derived concept.

In the same way, a role path $r=r_{1} \ldots r_{n}$ can be viewed as a derived role (denoted by role $(r))$ connecting instances of concept source $\left(r_{1}\right)$ to instances of concept target $\left(r_{n}\right)$. Similarly to derived roles, we can define derived attributes, by a role path followed by an attribute. Like attributes, these do not have inverses. Clearly, every role (attribute) is also a derived role (attribute).

Let $p=c . r$ be a concept path, $q$ be a prefix of $r$, and $q \backslash r$ denote $r$ with $q$ removed. If $c^{\prime}$ is either target (c.q) or a superconcept thereof but a subconcept of $\operatorname{source}(q \backslash r)$, then $c^{\prime} \cdot(q \backslash r)$ is called a suffix of $c . r$. Obviously, target $(p)$ is a suffix of $p$.

Given $D B$, a database of $\mathcal{S}$, we can associate extents with derived concepts in a straightforward manner. We note the following facts concerning these extents. First, the extent of $\operatorname{conc}(p)$ is a subset of the extent of $\operatorname{target}(p)$, hence also of its superconcepts in $\mathcal{S}$. Second, the extent of $\operatorname{conc}(p)$ is a subset of the extent of each of its suffixes. For example, it is easy to see that $p^{\prime}=$ Activity.produced is a suffix of $p=$ Person.carried_out.produced and all instances of $p$ (objects produced by an activity carried out by a person) are instances of $p^{\prime}$ (objects produced by activities).

Given a global schema $\mathcal{S}$, the derived schema (or extended schema) $\mathcal{S}_{\mathcal{X}}=$ $\left(C_{\mathcal{X}}, R_{\mathcal{X}}, A_{\mathcal{X}}\right.$, source,target, isa $\left._{\mathcal{X}}\right)$ is defined as follows : (i) $C_{\mathcal{X}}$ is the set of all derived concepts, defined by the concept paths definable in $\mathcal{S}$; (ii) $R_{\mathcal{X}}\left(A_{\mathcal{X}}\right.$ ) is the set of all the derived roles (attributes) defined by the role (attribute) paths definable in $\mathcal{S}$, and source and target are defined as above; (iii) the $i s a_{\mathcal{X}}$ relation contains the $i s a$ relations from $\mathcal{S}$, and additionally each pair $c, c^{\prime}$, where $c$ is a derived concept defined by a concept path $p$ in $\mathcal{S}$, and $c^{\prime}$ is the derived concept defined by a suffix of $p$.

Our interest in the derived schema is motivated by the fact that some sources may provide data only for derived concepts. The is a relationships in the derived schema enable us to use these sources to provide answers in terms of the original concepts. For example, even if a source provides only information about Person.carried sut.produced, this allows us to obtain some instances of Man_Made_Object, although not necessarily all. Note that answers obtained from sources in the local as view approach are partial answers in any case.

\subsection{Mapping Rules}

A source is integrated to the system, by providing a set of mapping rules that describe the relationships between the source schema and the global schema. There exist different ways for defining such views varying in terms of size and preciseness of the definition but also in the complexity of the query rewriting algorithm [7]. We have chosen 
essentially the same approach as in [7], namely to associate paths in the global schema with paths in the source schemas. This allows us to both associate concepts with XML nodes in the sources, and to associate relationships among concepts (expressed as roles or derived roles in the global schema) with XPath location paths in the XML sources.

Paths in a source are described in terms of XPath [6] location paths. We assume familiarity with the XPath language. Described in a nutshell, an XPath location path is composed of a sequence of location steps. Location steps have three parts: (i) an axis specifies the relationship (child, descendant, ancestor, attribute etc.) between the nodes selected by the location step and the context node, (ii) a node test specifies a node's XML type (element, attribute, and so on) and possibly its name, and (iii) optional predicates which use XPath expressions to further refine the set of selected nodes.

Let $V$ be a set of variables, and $U$ be a set of URLs. A mapping rule is an expression of the form $R: u / q$ as $v \rightarrow p$, where : (i) $R$ is the rule's label; (ii) $u \in V \cup U$, the rule's root, is either a variable or a URL ( $u$ is called the root of $R$ ); (iii) $q$ is an XPath location path, called the location path of the rule; (iv) as $v$ is a binding of $v$ ( $R$ is called the binding rule of $v$ ), where $v \in V$ is a variable; (v) $p$ is a schema path. More precisely, it is a role path if $u$ is a variable and a concept path otherwise. A rule $R$ is called a relative mapping rule if its root is a variable $u$, and an absolute mapping rule otherwise. In the first case, $u$ is the root variable of $R$, and this occurrence of $u$ is a use of the variable. Let $l p(R), s p(R)$ denote $R$ 's location path and schema path, respectively.

Given a set of mapping rules for a source $s$, we define reachability (in $s$ ) for rules and variables, as follows : (1) each rule whose root is a URL (the URL of $s$ ) is reachable; (2) each variable bound by a reachable rule is reachable; (3) finally, each rule whose root is a reachable variable is reachable. The set of mapping rules is cyclic if this definition of reachability leads to a cycle. The simplest case of a cycle is a rule whose left-handside contains $v / A$ as $v$ (provided that $v$ can be reached from a URL by other rules). In this work we consider only acyclic mappings.

A mapping $M$ over $\mathcal{S}_{\mathcal{X}}$ and for a source $s$ is a set of mapping rules such that 1) labels are unique (that is, no two rules have the same label), 2) all rules and variables are reachable in $M, 3$ ) the concepts, roles and attributes used in its rules occur in $\mathcal{S}_{\mathcal{X}}$ and 4) it contains no cycles.

The concatenation of mapping rules is defined as follows : two rules $R_{1}$ : $a / q_{1}$ as $v_{1} \rightarrow p_{1}, R_{2}: v_{1} / q_{2}$ as $v_{2} \rightarrow p_{2}$, can be concatenated, if the composition of their schema paths, $p_{1} \circ p_{2}$ is well defined ${ }^{5}$. Note the constraint that the root of $R_{2}$ is bound in $R_{1}$ and that concatenation is possible only if $p_{2}$ is a role path. The result of the concatenation is the rule $R_{1} \cdot R_{2}: a / q_{1} / q_{2}$ as $v_{2} \rightarrow p_{1} \circ p_{2}$.

Given a mapping $M$, its closure is the set of all rules that can be obtained from $M$ by repeated concatenation. It is denoted by $M^{*}$. Its expansion, denoted $\hat{M}$, is the set of absolute rules in $M^{*}\left(\hat{M} \subseteq M^{*}\right)$ and can be computed by a bottom-up fixpoint computation (since we only consider acyclic mappings, we are sure that a finite fixpoint exists).

Given a global schema $\mathcal{S}$, a mapping $M$ over $\mathcal{S}$ can naturally be interpreted in the derived schema $\mathcal{S}_{\mathcal{X}}$. Each absolute rule in $\hat{M}$ defines a derived concept, and each

\footnotetext{
${ }^{5}$ We do not define any restriction on the concatenation of the rules' location paths.
} 
relative rule in $M^{*}$ defines a derived role (attribute). Let us denote by $\mathcal{S}_{M}$ the restriction of $\mathcal{S}_{\mathcal{X}}$ to the derived concepts, roles and attributes of $M^{*}$.

For example, rule $R_{1} \cdot R_{3}$ defines a derived concept, conc(Person.carried ـut.produced) subconcept of Man_Made_Object, and rule $R_{3}$ defines a derived role, role(carried_out.produced), between concept Person and concept Man Made Object. Rule $R_{3} \cdot R_{4}$ defines a derived attribute attr(carried_out.produced.has _title) of concept Person.

A mapping $M$ for a source $s$ associated with URL $u$, allows us to view a collection of XML fragments reachable from $u$ as a database that conforms to $\mathcal{S}_{M}$. To define this database, the population of each derived concept, $\operatorname{conc}(p)$, is defined as the union of the set of fragments returned by all absolute rules $R$ in $\hat{M}$ where $s p(R)=p$ or $p$ is a suffix of $s p(R)$.

The set of fragments $X_{R}$ returned by some absolute rule $R$ in $\hat{M}$ is defined as follows. The root of an absolute rule $R$ is the URL $u$. Hence $X_{R}$ is assigned the set of XML fragments that can be obtained by applying the location path $l p(R)$ to the XML document identified by $u$. The set $X_{R}$ can be computed by a simple fixpoint computation, using rules in $M$. Since $M^{*}$ is finite, alternatively the rules of $\hat{M}$ can be used directly.

Similarly, the relative rules of $M^{*}$ are interpreted as roles (or attributes) of $\mathcal{S}_{M}$ in this database of XML fragments, represented by location paths.

Before leaving this subject, we note that according to the LAV approach, XML extents defined as above for the concepts are viewed as subsets of the real (but unknown) extents. Indeed, as sources are added, and rules are added to a mapping, the extents grow. In the LAV approach, any set of answers returned for a query is assumed to be a subset of the full (but unknown) answer.

\subsection{Keys}

As illustrated in Section 2, keys are essential to decide whether two XML fragments describe the same concept. We assume that sources are heterogeneous and autonomous, and we do not expect that they provide us with persistent object identifiers that are valid for all sources. The ID/ IDREF XML attribute mechanisms are used for internal references, but cannot serve for a key mechanism to perform joins between objects that originate from different sources. Sources might specify meaningful keys in terms of XML elements/attributes as proposed in $[4,8,16]$, but one cannot expect different autonomous sources to always use the same keys. For example a painting might be identified by its title in one source, by its title and the year of creation in another source.

A way to overcome this problem is to define global keys for concepts in the global schema. A key for a concept $c$ is defined as a list of derived attributes (called key paths) that originate from concept $c$ and is denoted by $k e y(c)=\left\{a_{1}, a_{2}, \ldots a_{n}\right\}$. W.1.g we assume in this paper that a concept is associated with at most one key, and all its subconcepts (including the derived ones) share the same key.

In our global schema, we could state for example, that an instance of concept Person is identified by attribute has name: $k e y$ (Person) $=\{$ has name $\}$. Instances of concept Man_Made_Object are identifiable by their title and their year of creation: 
key $($ Man_Made_Object $)=\{$ has_title, created_by $\cdot$ date.year $\}$. Images have no key, i.e. $\operatorname{key}(\operatorname{Image})=\emptyset$.

\section{Query Processing}

Our query processing approach is presented in this section. We first introduce the user query language (section 4.1 ). Two query processing strategies are then discussed. In the first approach (section 4.2), the solution to a query is the union of the complete answers from individual sources. If no complete answer can be obtained from a source, then the source is abandoned. In contrast, the second approach (section 4.3) allows also for incomplete answers from a given source. If a source $s$ can only partially answer a query, then the query is decomposed in two parts one to be fully answered by $s$ and the other part being sent to the other sources. The partial results from different sources are then joined by the mediator using global keys.

\subsection{Query Language}

The users query the virtual database as presented via the global schema, using simple tree queries, based on select-from-where clauses following an OQL-like syntax. Queries are of the form:

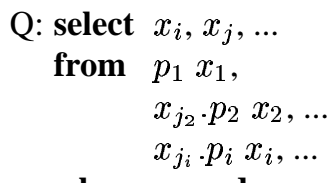

where $c_{0}$ and $c_{1}$ and ...

The $x_{i}$ 's are query variables and each $p_{i}$ in the from clause is a path in the global schema (schema path), called the binding path of $x_{i}$ and denoted $b p\left(x_{i}\right)$. The first variable $x_{1}$ is the root variable of the query, and its binding path $p_{1}$ is a concept path. For each $i>1$, there is a single clause $x_{j_{i}} \cdot p_{i} x_{i}$, and $p_{i}$ is a role path. We call $x_{j_{i}}$ the parent of $x_{i}$. We assume the parenthood relation between variables forms a tree, with $x_{1}$ as its root. $x_{1}$ ranges over the extent of the derived concept $\operatorname{conc}\left(p_{1}\right)$, and $x_{i}, i>1$, ranges over the instances defined by traversing instances of the derived role $p_{i}$ from the instances of its parent.

We assume queries satisfy the following restrictions. First, no restructuring is allowed in the select clause. Although this may add expressive power to the language, we feel it is not strictly needed for our application. Certainly, it is orthogonal to the issue of retrieving data from sources, addressed in this paper. Second, the where clause is a conjunction of simple predicates, where a simple predicate is of the form $x_{i} \theta d$ in which $\theta \in\{=,<,>, \leq, \geq\}$ and $d$ is an atomic value. Thus, it is not possible to express joins by equalities between variables, i.e., by predicates of the form $x_{i}=x_{j}$. This restricts the expressive power of the query language but simplifies the rewriting and evaluation of queries. Third, schema paths occur in the from clause, but not in the select clause or the where clause of a query. It is easy to show that a query with schema paths in the select and the where clause can be rewritten into an equivalent query in which they appear 
only in the from clause. Last, the language has no quantifiers, aggregates, or subqueries. However, a variable $x_{j}$ present in the from clause is implicitly existentially quantified. Thus, queries with certain kinds of existential quantification can be expressed in the above form.

Since no joins are allowed in the where clause, a query whose variables form a forest can be decomposed into a cross product of several tree queries: the restriction to tree rather than forest queries results in no loss of expressive power.

The result of a such a query is a set of tuples of the form $\left\{\left[a_{i}, a_{j}, \ldots a_{k}\right]\right\}$ where $a_{i}, a_{j}, \ldots a_{k}$ are instances of the variables in the query's select clause and can be either atomic values, or XML fragments.

In the sequel, the following representation of tree queries is used. A tree query $Q$ is represented as a labeled tree, $T(Q)=(X, p a r, b p, o p s)$ where $X$ is the set of query variables (tree nodes), par is the parent binary relation between nodes defined above, $b p(x)$ is the binding path of $x$ and ops is a set of operations associated with variable $x$, defined as follows : for a variable $x$ in the select clause $\pi \in$ ops $(x)$, and for each condition $x \theta d$ in the where clause, $\sigma_{x \theta d} \in$ ops $(x)$.

\subsection{Variable to Rules Bindings}

We now proceed to the details of query processing. We first present a simple approach in which a source contributes to the answer only if it can fully answer the query.

To evaluate a query, we need to rewrite it into an XML query that some sources can answer. Obviously, in general only some of the sources contain the data requested in the query. Each such source returns a subset of the possible answers; the union of the answers from all relevant sources is presented to the user (see Section 5).

For this rewriting, we use the mapping rules. For a query $Q$ and a source $s$, we define a variable to rule binding, or shortly variable binding, as a mapping $\beta$ from a set of query variables to $M^{\star}$. We consider only bindings such that $\operatorname{dom}(\beta)$ is either empty or is the set of nodes of some prefix ${ }^{6}$ of $T(Q)$. The empty binding is denoted by $\beta_{\emptyset}$. If $\beta$ binds all variables in $Q$ then it is called a full binding, otherwise it is a partial binding.

The properties of a binding $\beta$ are the following : if $\operatorname{dom}(\beta)$ is not empty, then $\beta$ associates each variable in it with a rule of $M^{*}$, such that the following hold:

1. if $x$ is the root of query $Q$, then $\beta(x)$ is an absolute mapping rule such that $\operatorname{conc}(s p(\beta(x))) i s a_{M} \operatorname{conc}(b p(x))$, i.e., the derived concept defined by $s p(\beta(x))$ is a subconcept of the derived concept defined by the binding path $b p(x)$ in $\mathcal{S}_{M}$,

2. else, let $\operatorname{par}(x)=x^{\prime}$, then $\beta(x)$ is a relative rule, and

- the root variable of rule $\beta(x)$ is bound in rule $\beta\left(x^{\prime}\right)$,

- the role path (RHS) of the rule $\beta(x)$ is equal to the binding path $b p(x)$,

- and finally, the concatenation of the two rules $\beta\left(x^{\prime}\right)$ and $\beta(x)$ is well-defined.

In the first case, $x$ is the root of $Q$ and bound to some (possibly derived) concept by its binding path $b p(x)$ that has the form $c$ or $c . r$. An absolute rule $R$ can provide instances for this concept if its concept path (RHS) $s p(R)$, viewed as a derived concept, is a sub-concept of $b p(x)$ (i.e. if the latter is a suffix of $s p(R)$ or it defines a superconcept

\footnotetext{
${ }^{6}$ A tree $T^{\prime}$ is a prefix of a tree $T$ if it is a subtree of $T$ and its root is the same as that of $T$.
} 
thereof). Note that we use here both derived concepts and the isa relationship between them. Thus, the derived schema defined in Section 3 is essential for our approach to query processing.

In the second case, the assumption that if $\beta$ is defined on $x$ then it is defined on the parent of $x$ follows from the requirement that its domain is a prefix of $T(Q)$. In this case, the declaration of $x$ in $Q$ has the form $x^{\prime} \cdot q x$, and $b p(x)=q$. Answers for $x$ can be obtained from answers for $x^{\prime}$, by following the binding path $q$ of $x$.

A partial binding $\beta$ is called maximal if there does not exist a binding $\beta^{\prime}$ such that $\operatorname{dom}(\beta) \subset \operatorname{dom}\left(\beta^{\prime}\right)$ and $\beta(x)=\beta^{\prime}(x)$ for all $x$ in $\operatorname{dom}(\beta)$. It is evident that a full binding is a maximal binding.

Variable Binding Algorithm We will now describe a variable binding algorithm $\mathcal{B}(Q, s)$ which takes as an input a query $Q$ and a mapping $M$ for a source $s$ and returns a set of maximal bindings. A binding $\beta$ is represented as a vector of associations of variables to rules, $\left[x_{1} \mapsto R_{1}, \ldots x_{n} \mapsto R_{n}\right]$. The algorithm is illustrated in more detail in Fig. 6. First, the variables of the query tree are arranged in pre-order: the root is first, and every other node occurs after its parent. The algorithm starts from the empty binding, and once a set of partial bindings have been constructed, it tries to extend each one, using the ordering of the variables. The extension of a partial binding $\beta$ by $[x \mapsto R]$ is denoted $\beta \times[x \mapsto R]$.

In the first step, we extend $\beta_{\emptyset}$ to the root variable $x_{1}$. For each absolute rule $R$ in $M^{*}$ such that the derived concept defined by $s p(R)$ is a subconcept of the derived concept defined by the concept path $b p\left(x_{1}\right)$ in $\mathcal{S}_{M}$, we create the binding $\left[x_{1} \mapsto R\right]$ and add it to the set of bindings for $x_{1}$. If no absolute rule is found such that the above conditions hold, then the algorithm stops, and returns the empty set. Then, we iterate through the sequence of variables, from the left. Let the current, not yet treated, variable be $x_{i}$, and let $y$ be its parent. For each binding $\beta$ constructed so far, if $y \notin \operatorname{dom}(\beta)$ then $\beta$ cannot be extended to $x_{i}$ (recall that a binding is always defined on a prefix of $T(Q)$ ). Else, let binding $\beta$ associate rule $R^{\prime}$ with $y$. Then, for each relative rule $R$ of $M^{*}$, such that $b p\left(x_{i}\right)=s p(R)$, if $R$ and $R^{\prime}$ can be concatenated (i.e, $R^{\prime}$ binds the variable that is the root of $R$, and their schema paths can be composed), we extend $\beta$ by $\left[x_{i} \mapsto R\right]$. In this case, $\beta$ can be dropped, since the new binding extends it. Note that the edge from $y$ to $x_{i}$ is traversed in this step, and only in this step.

$\mathcal{B}(Q, s)$ finds the maximal bindings for a query $Q$ and a mapping $M$ on source $s$. The proof is straightforward. Consider a binding $\beta$ in the result set of $\mathcal{B}(Q, s)$. If there exists a variable $x$ that we could add in $\operatorname{dom}(\beta)$, this means that there exists some rule $R$ such that $\beta$ (parent $(x))$. $R$ is well-defined, then by the algorithm $x$ would already be in $\operatorname{dom}(\beta)$ which is a contradiction, from the above assumption.

Let us illustrate the algorithm with query $Q_{2}$ presented earlier, and the mapping rules for source $S_{1}$ illustrated in Fig. 4. Rule $R_{1}$ returns answers for variable $a$. The rule's schema path is Person which is equal to $a$ 's binding path (Person). Rule $R_{2}$ returns answers for $b$, since (1) its schema path has name is equal to the variable's binding path, (2) its root variable $u_{1}$ is bound in rule $R_{1}$, and (3) the composition of the schema paths of rules $R_{1}$ and $R_{2}$ is well defined, since attribute has name is defined in concept Person. In a similar manner, we find that variable $c$ is bound to rule $R_{3}$ and 


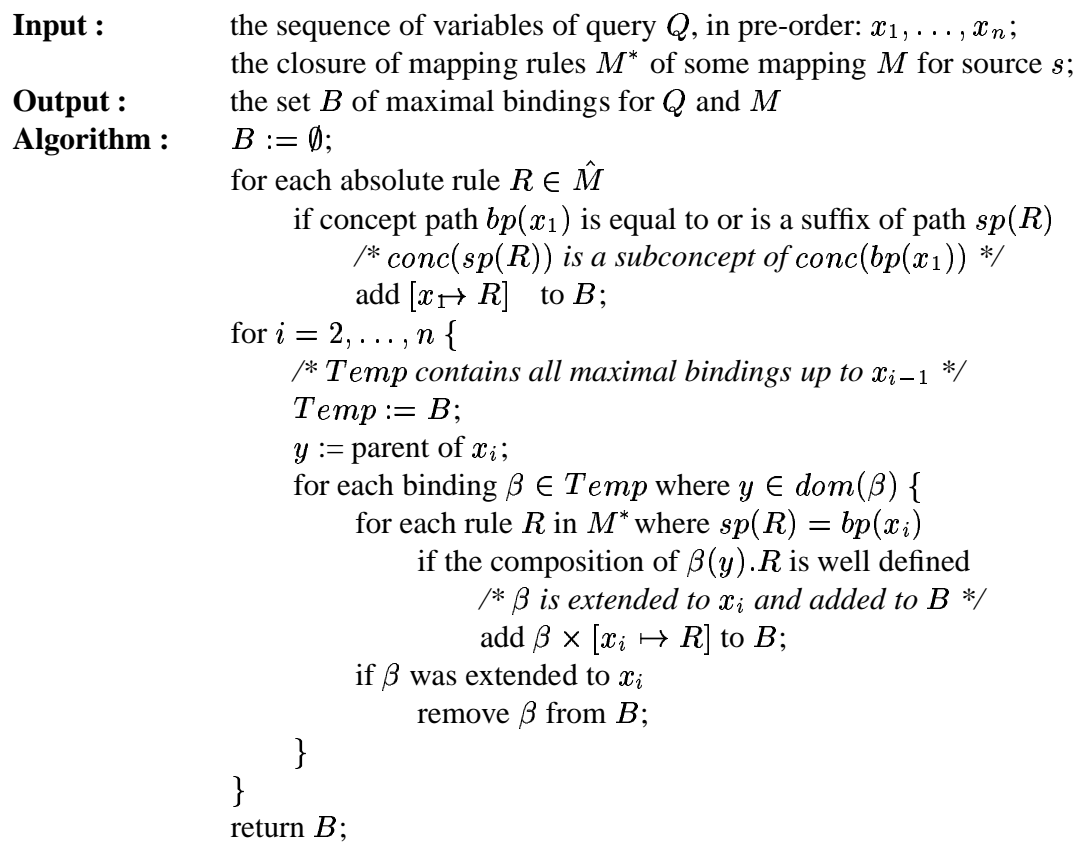

Fig. 6. Variable binding Algorithm $\mathcal{B}(Q, s)$

variable $d$ to rule $R_{4}$. For variable $e$, we do not find a mapping rule whose schema path is equal to the variable's binding path (located at). The result $\mathcal{B}\left(Q_{2}, S_{1}\right)$ is the singleton $\left\{\beta_{1}\right\}=\left\{\left[a \mapsto R_{1}, b \mapsto R_{2}, c \mapsto R_{3}, d \mapsto R_{4}\right]\right\}$.

\subsection{Query Decomposition}

Let $S$ be the set of sources mapped to the global schema. Algorithm $\mathcal{B}(Q, s)$ returns for each source $s$ in $S$ the set of maximal bindings. Each such binding $\beta$ is either full, i.e. $\operatorname{dom}(\beta)$ contains all variables in $Q$ (then $s$ can answer the query using $\beta$ ), or partial. In the latter case, $\beta$ provides us with partial answers, i.e. does not provide answers for all variables in the query. To complete these partial answers, we decompose the query $Q$ into (i) a prefix query that source $s$ can answer using binding $\beta$, denoted $Q_{p}(\beta)$, and (ii) a set of suffix queries, denoted $\mathcal{Q S}(\beta)$.

As an example, take the result of algorithm $\mathcal{B}\left(Q_{2}, S_{1}\right)$ calculated for query $Q_{2}$, and source $S_{1}$ published by the mapping rules illustrated in Fig. 4. It contains a partial binding $\beta_{1}$ defined on a proper subset of the variables in the initial query: for an instance of variable $c$ we miss instances for variable $e$ (and its descendants).

To obtain the complete answer, we define (1) a prefix query that source $S_{1}$ can answer using $\beta$ (query $Q_{p}\left(\beta_{1}\right)=Q_{2}(a)$ illustrated in Fig. 5) and one suffix query (query $Q_{2}(b)$ illustrated in Fig. 5). The prefix query $Q_{p}(\beta)$ is a prefix of $T(Q)$ and is defined on the set of variables in $\operatorname{dom}(\beta)$. The suffix queries of a prefix $Q_{p}(\beta)$ in $Q$ 
are defined as follows. Let $N$ be the set of variables in $Q_{p}(\beta)$ which contain at least one child in $Q$ but not in $Q_{p}(\beta)$ (we call $N$ the boundary of $Q_{p}(\beta)$ ). Then we define a suffix query for each variable $x$ in $N$ as the subtree of $Q$ rooted at $x$ and containing all descendants of $x$ not in $Q_{p}(\beta)$. It is easy to see that query $Q_{2}(b)$ illustrated in Fig. 5 is a suffix query of $Q_{2}(a)$ in $Q_{2}$. Observe that for a given prefix query there might exist zero, one or more suffix queries.

Joining the results The results of the prefix query and the suffix queries must be joined. In order to perform the join between a prefix and a suffix query the following two conditions must hold: (1) the concept to which the root of a suffix query is bound, should have a key, and (2) the sources on which the join is performed should provide complete values for this key.

The key values of an instance of a concept $c$ are obtained by considering $k e y(c)$ as a query ranging over all key paths in it. The result of a key query is of the form $\left\{\left[x_{1}, x_{2}, . ., x_{n}\right]\right\}$, where the $x_{i}^{\prime} s$ are instances of the variables to which the key paths are bound in the key query. For example, the query illustrated below returns the key values for an instance $x$ of concept Man_Made_Object :

key(Man_Made_Object): select $t, y$

from Man_Made_Object $x$,

$x$.has_title $t, x$.created_by.date.year $y$

Given a (prefix or suffix) query $Q$ whose result is of the form $\left\{\left[a_{1}, a_{2}, \ldots a_{n}\right]\right\}$ where the $a_{i}^{\prime} s$ are instances of the variables in the query's select clause and a key query $\operatorname{key}(c)$, where $c$ is the concept on which the join will be performed, $Q$ is extended to $Q^{\prime}$ so as to get, as well as the $a_{i}$ 's, the key values for the fragments, instances of concept $c$, accessed by $Q$. The result of $Q^{\prime}$ is of the form $\left\{\left[a_{1}, a_{2}, \ldots a_{n}, x_{1}, x_{2}, \ldots, x_{p}\right]\right\}$ where the $x_{j}$ 's are instances of the key query variables (variables bound to the key paths in $k e y(c)$ ). For example, the prefix query obtained after extending query $Q_{2}(a)$ of Fig. 5 by the key of concept Man_Made_Object is given below ${ }^{7}$.

$\operatorname{ext}\left(Q_{2}(a)\right)$ : select $d, t, y$

from Person $a, a$.has_name $b$, a.carried_out.produced $c, c$.has title $d$, $c$.has_title $t, c$.created_by.date.year $y$ where $b=$ "Van Gogh"

Query Execution Plans Let $Q$ be a query and $S$ be a set of sources. A decomposition of $Q$ w.r.t. some maximal binding $\beta$ is a couple $\mathcal{D}(Q, \beta)=\left[Q_{p}(\beta), \mathcal{Q S}(\beta)\right]$ such that $Q_{p}(\beta)$ is a prefix query of $Q$ on source $s$ in $S$ and $\mathcal{Q S}(\beta)$ is the set of suffix queries of $Q_{p}(\beta)$ in $Q$. For example, $\mathcal{D}\left(Q, \beta_{1}\right)=\left[Q_{2}(a),\left\{Q_{2}(b)\right\}\right]$ is a decomposition for query $Q_{2}$ and the maximal binding $\beta_{1}$ defined on source $S_{1}$. Observe that $\mathcal{Q S}(\beta)$ is empty if $\beta$ is a full binding for $Q$.

Let $\mathcal{D}(Q, \beta)=\left[Q_{p}(\beta), \mathcal{Q S}(\beta)\right]$ be a decomposition of $Q$. Then $Q_{p}(\beta)$ can be translated into a source query using binding $\beta$. For each suffix query in $\mathcal{Q S}(\beta)$ either

\footnotetext{
${ }^{7}$ This query can be optimized by keeping the variables that are common to the key query and the prefix query (the case of variables $d$ and $t$ in the example above).
} 
a full binding is found or the suffix query has still to be decomposed. Let $Q_{i}$ be a suffix query in $\mathcal{Q S}(\beta)$ and $\boldsymbol{k}_{\boldsymbol{i}}=\left[x_{1}, \ldots x_{p}\right]$ denote the key query variables bound to the key paths of concept $c_{j}$ associated to the root variable of $Q_{i}$. Then $Q_{p}(\beta) \nabla_{\boldsymbol{k}_{\boldsymbol{i}}} Q_{i}$ denotes the join operation between $Q_{p}(\beta)$ and $Q_{i}$ (we assume that both queries are extended by the appropriate key queries). A prefix query rewriting $\mathcal{L} \mathcal{R}(Q, \beta)$ for a decomposition $\mathcal{D}(Q, \beta)$ is defined as the join between a prefix query and all suffix queries $Q_{i}, 1 \leq i \leq n$, in $\mathcal{Q S}(\beta)$ (if $\beta$ is a full binding for $Q$ then $\mathcal{Q S}(\beta)$ is empty and $\left.\mathcal{L} \mathcal{R}(Q, \beta)=Q_{p}(\beta)\right)$ :

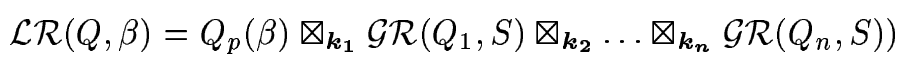

Then the initial query $Q$ can be rewritten as $\mathcal{G R}(\mathrm{Q}, \mathrm{S})$ defined as the union of all prefix rewritings for sources in $S$ :

$$
\mathcal{G R}(Q, S)=\bigcup_{s \in S} \bigcup_{\beta \in \mathcal{B}(Q, s)} \mathcal{L} \mathcal{R}(Q, \beta)
$$

Let a query execution plan (QEP) be defined as follows : (1) a query $q$ that can be answered by a single source (that is a query for which there exists a full binding) is a(n atomic) QEP; (2) the union of two QEP's is a QEP ${ }^{8}$; (3) the join of two QEP's is a $\mathrm{QEP}^{9}$. Basically, sources answer atomic queries in a QEP and the mediator performs joins and unions. A QEP can involve several atomic queries sent to a given source. It might be interesting to combine such queries in a single query. This implies the reorganization using classical properties such as distributivity of union w.r.t. join. Such properties and reorganizations as well as other optimizations are beyond the scope of this paper.

Given a set of sources $S$ and a query $Q$, the algorithm $P(\mathrm{Q})$ shown in Fig. 7 computes a query execution plan for $Q$. For each source $s$ and maximal binding $\beta \in \mathcal{B}(Q, s)$, a QEP $P(\beta)$ of the prefix rewriting $\mathcal{L} \mathcal{R}(Q, \beta)$ is computed: if $\beta$ is a full binding (i.e. complete answers are obtained), the result is query $Q$. Else, if $\beta$ is a partial binding, then query $Q$ is decomposed into a prefix query $Q_{p}(\beta)$ and a set of suffix queries $\mathcal{Q S}(\beta)$ (these queries are also extended by the key queries as shown before). The query execution plan of $Q$ against source $s$ is obtained by joining $Q_{p}(\beta)$ with the query execution plan for each suffix query $Q^{\prime} \in \mathcal{Q S}(\beta)$ (variable $k^{\prime}$ denotes the key query variables of $Q^{\prime}$ ). To calculate the query execution plan of a suffix query $Q^{\prime}$ the algorithm is called recursively. Finally the obtained plan is added to the existing plan by union.

Observe that there are two reasons to interrupt the calculation of a query execution plan for a given source $s$ and binding $\beta$. The most trivial case is that there exists no maximal binding for $Q$ in $s$. The second reason is that there exists at least one suffix query which cannot be satisfied (empty query execution plan).

\footnotetext{
${ }^{8}$ Remember that union is heterogeneous, that is two sets of tuples answering the same query but resulting from different sources might have different structures for the $i$-th component.

${ }^{9}$ We restrict join to the non commutative aforementioned definition of join: the root of the second QEP should belong to the boundary of the first QEP and each of them should correspond to a concept for which a key has been defined.
} 


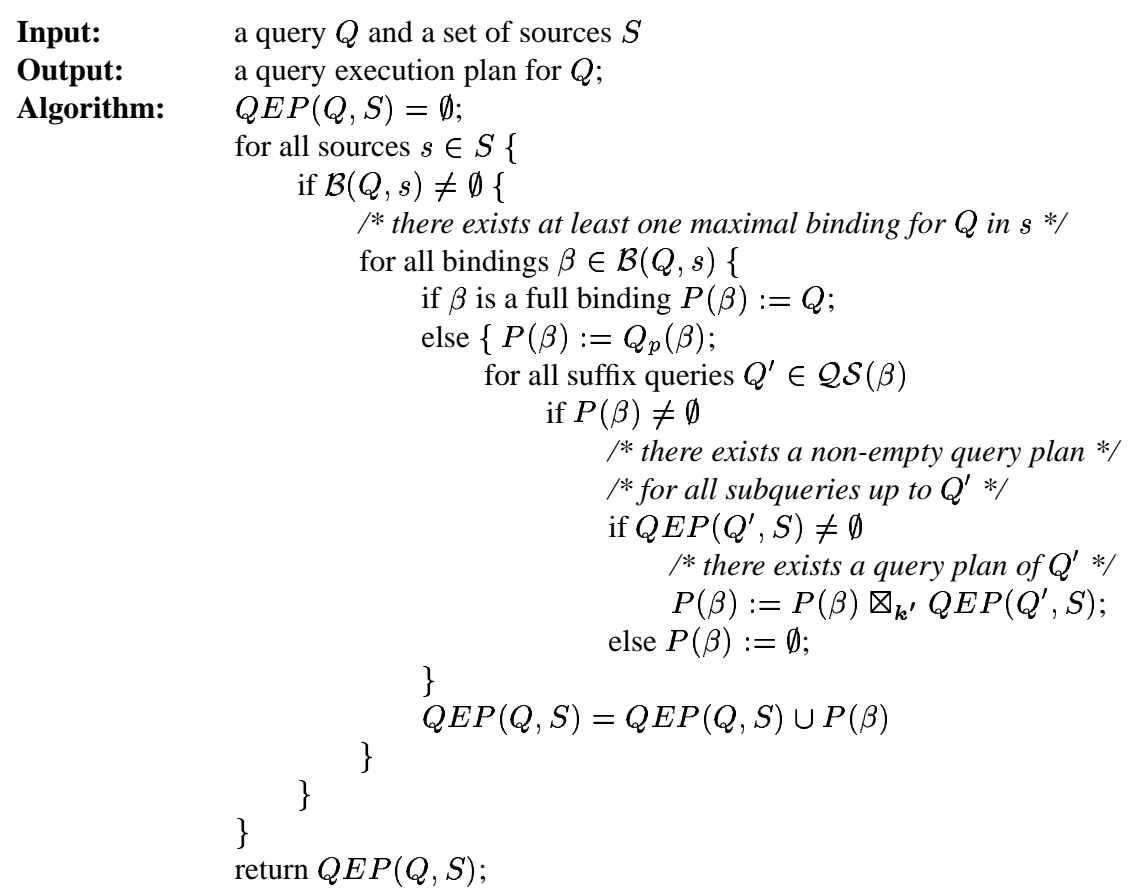

Fig. 7. Query Execution Plans Generation

\section{System Architecture}

In this section we sketch the architecture of the prototype $S T_{Y} X$ [9] (Fig. 8) that implements the data integration approach described previously. XML Web resources can be published on the fly by creating/modifying/deleting mapping rules between source fragments and the global schema using the Source Publication Interface. The global schema can be consulted through the Schema Manager which is also responsible for its loading in a $S T_{Y} X$ portal. The mapping rules are first validated by the Rules Manager which is also responsible for their storage. The publication of a resource also consists in providing an XSLT transformation program ${ }^{10}$ that can be used for formatting source data in the query result ${ }^{11}$. Query processing is done in several steps: first user queries can be formulated using a standard Web browser. They are either created by a generic Query Interface, or simply stored in the form of a hypertext link (URL). The Query Interface communicates with the Schema Manager allowing the user to browse the global schema for the formulation of a query. The Query Interface forwards the query to the Query Parser which performs a syntactical analysis of the query with some typechecking w.r.t. the global schema and produces a language neutral intermediate repre-

\footnotetext{
${ }^{10}$ XSL Transformations (XSLT : http://www.w3c.org/TR/xslt)

${ }^{11}$ If the query result contains XML fragments from a source, then those are transformed using the source's XSL Stylesheet.
} 


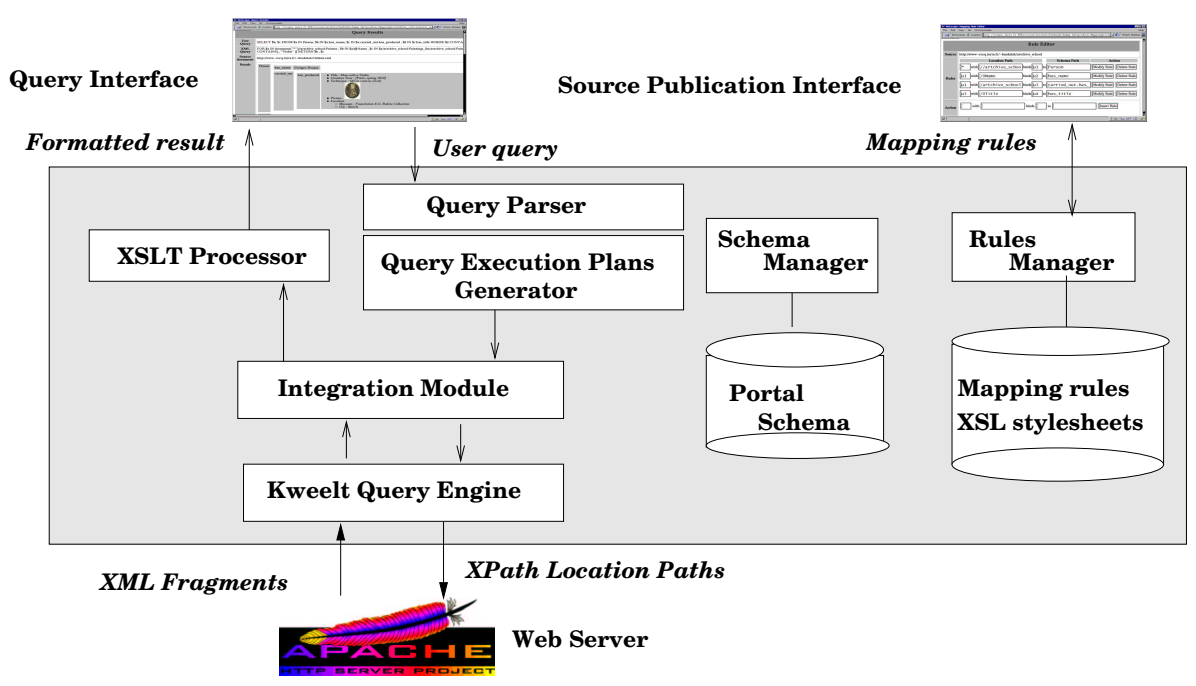

Fig. 8. $S T_{Y} X$ Portal Architecture

sentation of it. The query is then forwarded to the Query Execution Plans Generator, which creates the query execution plan. The Integration Module rewrites the queries into Quilt Queries and sends them to the Kweelt Query Engine ${ }^{12}$ for evaluation ${ }^{13}$. The resulting XML fragments are sent to the Integration Module that combines the results. This module, based on the query and the mapping rules, inserts schema specific tags, and then the XSLT Processor (Cocoon ${ }^{14}$ ) finally transforms the result into an HTML document which is displayed to the browser of the user.

The $S T_{Y} X$ prototype was implemented in Java JDK 1.2. XML technologies such as XSLT, XPath and the Xalan XML Stylesheet processor ${ }^{15}$ were used.

\section{Related Work}

Data integration has become an important issue during the past years and a large number of integration systems have been proposed. These systems can be classified according to the architectures used for query processing : data warehouse systems materialize all source data before query processing, whereas mediators propose a virtual database and push queries to the source level based on sophisticated query rewriting algorithms. Our approach clearly belongs to the second category.

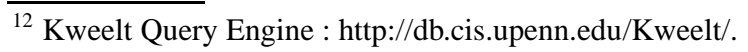

${ }^{13}$ The Kweelt query engine can evaluate the subset of XQuery expressions presented in this paper.

${ }^{14} \mathrm{http}: / / \mathrm{xml}$.apache.org/cocoon

${ }^{15} \mathrm{http} / / / \mathrm{xml}$.apache.org/xalan-j/index.html
} 
Mediator systems are classified according to the way sources are described to the mediator and queries are evaluated [11]. Tsimmis [14], MIX [3], YAT [5] and Picsel [10] follow the global as view approach and are not directly comparable to ours. On the other hand, Information Manifold [12] follows the local as view approach. In this system the global schema is a flat relational schema, and Description Logics is used to represent hierarchies of classes. The sources are expressed as relational views over this schema. Query rewriting is done by the Bucket algorithm which rewrites a conjunctive query expressed in terms of the global schema using the source views. It examines independently each of the query subgoals and tries to find rewritings but loses some by considering the subgoals in isolation. The MiniCon algorithm [15] improves the Bucket algorithm by exploiting the input/output dependencies between the query subgoals for reducing the search space of possible rewritings. Algorithm $\mathcal{B}(Q, S)$ presented in this paper resembles to MiniCon since it exploits the parent/child dependencies of query variables for query decomposition.

The Agora [13] system, offers an XML view for relational and XML data and user queries are XQuery expressions. Although XML is used as the global data model, an extended use of the relational model is made: the XML view is translated into a generic relational schema, XML resources are described as relational views over this schema and XQuery expressions are translated to standard SQL queries which are then decomposed, optimized and evaluated. Our system and query rewriting algorithm extensively exploit the tree structure of XML data which is described as local views of a more powerful conceptual schema with inheritance.

Last, the Xyleme [7] system is based on a data-warehouse solution for the integration of XML data ("all XML data of the Web"). However, it can be considered as a mediator system, since source data is stored without transformation and users can query this data via different views. Each view is described by a DTD, called abstract DTD, and source data is mapped to one or several DTDs using path-to-path mapping rules. These rules are similar to our mapping rules with the difference that they map absolute source paths (starting from the document root) to absolute paths in the abstract DTD (starting from the DTD root element).

\section{Conclusions}

We proposed in this paper an alternate approach for integrating XML sources following the LAV approach. Instead of choosing for the global view, a relational or XML schema, we advocated the use of an ontology-based mediation. The global schema is close to an object-oriented schema on a terminology describing a common domain of interest and users issue queries on this global schema. Our contributions are (i) a view definition language, (ii) a rewriting algorithm, (iii) an algorithm for generating execution plans, and (iv) a prototype validating the approach.

We are currently working on several extensions concerning our integration model. First, we try to extend the query language by allowing explicit joins in the where clause of a query. This does not change the binding algorithm, but increases the complexity of query processing. A second issue we are looking at concerns the usage of maximal bindings for query decomposition. In fact, the current version of the rewriting algorithm 
generates query execution plans which favor information stored locally in the same document. For example, if some source $s$ provides a single full binding for some query $Q$, the algorithm will return the result of $Q$ in $s$, but will not try to join $s$ with some other source $s^{\prime}$. This restriction can be removed by allowing also partial bindings that are not maximal, but will increase the number of possible decompositions significantly.

\section{References}

1. S. Abiteboul, P. Buneman, and D. Suciu. Data On the Web: From Relations to Semistructured Data and XML. Morgan Kaufmann, October 1999.

2. B. Amann, C. Beeri, I. Fundulaki, and M. Scholl. Ontology-Based Integration of XML Web Resources. In International Semantic Web Conference (ISWC), Sardinia, Italy, 2002.

3. C. Baru, A. Gupta, B. Ludäscher, R. Marciano, Y. Papakonstantinou, P. Velikhov, and V. Chu. XML-based information mediation with MIX. In Demonstrations, ACM/SIGMOD, pages 597-599, 1999.

4. P. Buneman, S. B. Davidson, W. Fan, C. S. Hara, and W. C. Tan. Keys for XML. In Proc. WWW10, pages 201-210, 2001.

5. V. Christophides, S. Cluet, and J. Simeon. On Wrapping Query Languages and Efficient XML Integration. In Proc. of ACM SIGMOD, Dallas, USA, May 2000.

6. J. Clark and S. DeRose (eds.). XML Path Language (XPath) Version 1.0. W3C Recommendation, November 1999. http://www.w3c.org/TR/xpath.

7. S. Cluet, P. Veltri, and D. Vodislav. Views in a Large Scale XML Repository. In Proc. VLDB, Rome, Italy, September 2001.

8. W. Fan, G. Kooper, and J. Simeon. A Unified Constraint Model for XML. In Proc. WWW10, Hong-Kong, China, May 2001.

9. I. Fundulaki, B. Amann, C. Beeri, and M. Scholl. STYX : Connecting the XML World to the World of Semantics. In Proceedings of EDBT, Prague, Czech Republic, March 2002. (Demonstration).

10. F. Goasdoué, V. Lattés, and M-C. Rousset. The use of CARIN language and algorithms for information integration: The PICSEL System. International Journal on Cooperative Information Systems, 2000.

11. A. Halevy. Theory of answering queries using views. SIGMOD Record, 29(4):40-47, 2000.

12. A. Levy, A. Rajaraman, and J. Ordille. Querying Heterogeneous Information Sources Using Source Descriptions. In Proc. VLDB, pages 251-262, Mumbai (Bombay), India, September 1996.

13. I. Manolescu, D. Florescu, and D. Kossmann. Answering XML Queries over Hterogeneous Data Sources. In Proc. VLDB, Rome, Italy, September 2001.

14. Y. Papakonstantinou, H. Garcia-Molina, and J. Widom. Object Exchange Across Heterogeneous Information Sources. In Proc. ICDE, pages 251-260, Taipei, Taiwan, March 1995.

15. R. Pottinger and A. Levy. A Scalable Algorithm for Answering Queries Using Views. In Proc. VLDB, pages 484-495, Cairo, Egypt, September 2000.

16. H. Thompson, D. Beech, M. Maloney, and N. Mendelsohn. XML Schema Part 1: Structures. W3C Recommendation, May 2001. http://www.w3.org/TR/XML-schema-1. 